\title{
Immortalization and characterization of pleomorphic adenoma cells by transfection with the $h T E R T$ gene
}

\author{
MASAE KITAGAWA ${ }^{1,2}$, IKUKO OGAWA ${ }^{2}$, KAORI SHIMA ${ }^{3}$, SADAMITSU HASHIMOTO $^{3,4}$, YASUSEI KUDO ${ }^{1}$, \\ MUTSUMI MIYAUCHI ${ }^{1}$, HIDETOSHI TAHARA ${ }^{5}$, MASAKI SHIMONO $^{3}$ and TAKASHI TAKATA ${ }^{1}$ \\ ${ }^{1}$ Department of Oral and Maxillofacial Pathobiology, Graduate School of Biomedical Sciences, Hiroshima University, \\ ${ }^{2}$ Center of Oral Clinical Examination, Hiroshima University Hospital, 1-2-3 Kasumi, Minami-ku, Hiroshima 734-8553; \\ ${ }^{3}$ Department of Pathology, and ${ }^{4}$ Oral Health Science Center, Tokyo Dental College, 1-2-2 Masago, Mihama-ku, \\ Chiba 261-8502; ${ }^{5}$ Department of Cell and Molecular Biology, Graduate School of Biomedical Sciences,
} Hiroshima University, 1-2-3 Kasumi, Minami-ku, Hiroshima 734-8553, Japan

Received March 13, 2007; Accepted May 4, 2007

\begin{abstract}
Pleomorphic adenomas (PAs) of salivary glands are characterized by the mixed appearance of epithelial and mesenchymal-like components such as myxoid and chondroid tissues. Although various studies have examined PAs, thus far it is not clear how PAs make these multiple components. Thus, clarification of the histodifferentiation of this unique salivary gland tumor using not only tissues in vivo but also PA cells cultured in vitro is necessary. However, no in vitro model of PA has been reported, because normal and benign tumor cells tend to grow slowly and senesce quickly in culture. Therefore, we immortalized cells using transfection of the hTERT gene without otherwise altering the nature of those cells. The immortalized PA cells expressed mRNA of the pleomorphic adenoma gene 1 and showed epithelial and neoplastic myoepithelial characteristics by immunohistochemical immunofluorescence analyses and ultrastructural study. Our findings suggest that these cells will be a useful model to study the cellular differentiation of PA.
\end{abstract}

\section{Introduction}

Pleomorphic adenomas (PAs) of the salivary glands are characterized by the mixed appearance of epithelial and mesenchymal-like components such as myxoid and chondroid tissues. Although various studies have examined PAs $(1,2)$, it is not clear how they make those multiple components. Thus, it is necessary to clarify the histodifferentiation of this unique type of salivary gland tumor using not only tissues in vivo but

Correspondence to: Dr Takashi Takata, Department of Oral and Maxillofacial Pathobiology, Division of Frontier Medical Science, Graduate School of Biomedical Sciences, Hiroshima University, 1-2-3 Kasumi, Minami-ku, Hiroshima 734-8553, Japan

E-mail: ttakata@hiroshima-u.ac.jp

Key words: pleomorphic adenoma, immortalization, hTERT also PA cells cultured in vitro. However, no in vitro cell model of PA has been reported, because normal and benign PA tumor cells tend to grow slowly and senesce quickly in culture.

Generally, the loss of DNA from the telomeres of chromosomes that occurs with each replication shortens them to a critical minimal length after which the cells senesce (3). The parental PA cells used in this study entered the crisis state after 6 population doublings (PDLs). Telomerase is a ribonucleoprotein reverse transcriptase that stabilizes the telomere and chromosome structure by adding telomeric repeats that would otherwise be lost during replication (4-8). The telomerase enzyme consists of three major components, human telomerase RNA (hTR), human telomerase-associated protein (hTLP1) and human telomerase reverse transcriptase (hTERT), the latter being the catalytic subunit of telomerase (9). Normal and neoplastic cells usually express hTR and hTLP1 (10), although most normal cells and benign neoplastic cells, including PA, do not possess telomerase activity (11-13). However, malignant tumor cells exhibiting telomerase activity express high levels of hTERT, which seems to be sufficient for cells to regain telomerase activity (11).

In the present study, to generate a stable and standard culture of human PA cells, we established immortalized human PA cells by infecting them with a retroviral vector containing hTERT. We describe the cellular phenotypic characteristics of these immortalized PA cells, which will be a useful model to study the cellular differentiation of PA.

\section{Materials and methods}

All procedures were performed in compliance with regulations administered by Hiroshima University.

Cells and culture conditions. Surgical tissue fragments were obtained from a PA of the parotid gland of a 74-year-old Japanese female patient at Hiroshima University Hospital. The tissue specimen from the central part of the tumor was cut into small pieces, placed in $90-\mathrm{mm}$ culture dishes with keratinocyte serum-free medium (Invitrogen, Grand Island, $\mathrm{NY}$ ), and incubated in $5 \% \mathrm{CO}_{2}$ in air at $37^{\circ} \mathrm{C}$. Outgrowing 
cells were then subcultured in the same medium and used for analysis.

Transfection. The hTERT gene was cloned into the retroviral vector, pQCXIP (Clontech, Tokyo, Japan) with the HpaI restriction enzyme. The cloned retrovirus was transfected into the RetroPack PT67 Packaging Cell Line (Clontech), where the vector was packaged into infectious, replicationincompetent retroviral particles. The packaged cells were maintained with puromycin $(1.2 \mu \mathrm{g} / \mathrm{ml})$ containing medium for 1 week. Retrovirus for hTERT was collected, polybrene was added $(8 \mu \mathrm{g} / \mathrm{ml})$ and the mixture was then filtered through a $0.20-\mu \mathrm{m}$ filter. After puromycin treatment for 1 week, packaging cells were maintained without puromycin. To infect target cells, the packaged retrovirus ( $1 \mathrm{ml} / \mathrm{dish}$ ) was added to the medium of a $25-\mathrm{mm}^{2}$ flask containing cultured target cells at $50-60 \%$ confluence. After $24 \mathrm{~h}$, infected cells were placed in medium containing puromycin $(0.5 \mu \mathrm{g} / \mathrm{ml})$ for 2 to 3 weeks.

$R N A$ preparation and reverse transcription-polymerase chain reaction analysis $(R T-P C R)$. Total RNA was isolated from cultures of confluent cells using the RNeasy Mini kit (Qiagen Inc., Valencia, CA) according to the manufacturer's instructions. Total RNA was quantified and its purity was determined by standard spectrophotometric methods. cDNA was synthesized from $1 \mu \mathrm{g}$ total RNA using ReverTra Dash (Toyobo Biochemicals, Tokyo, Japan). The oligonucleotide RT-PCR primers listed in Table I were purchased from Invitrogen (Carlsbad, CA). Aliquots of total cDNA were amplified with 1.25 U of rTaq-DNA polymerase (Qiagen Inc.), and amplifications were performed using a PC701 thermal cycler (Astec, Fukuoka, Japan) for 30 cycles after an initial 30-sec denaturation at $94^{\circ} \mathrm{C}$, annealed for $30 \mathrm{sec}$ at $56^{\circ} \mathrm{C}$, and extended for $1 \mathrm{~min}$ at $72^{\circ} \mathrm{C}$. The amplification reaction products were resolved on $1.5 \%$ agarose/TAE gels (Nacalai Tesque Inc., Kyoto, Japan), electrophoresed at $100 \mathrm{mV}$, and visualized by ethidium-bromide staining. The sequences of the primers used were as follows: hTERT primers, F 5'-ACTTTGTCAA GGTGGATGTGACGG-3', R 5'-AAGAATCATCCACCA AACGCAGG-3'; PA gene (PLAG)1 primers, F 5'-CAAGT GCAGCCAGCAATTTA-3', R 5'-CATTTCCCAGAGATG CATGA-3; and glyceraldehyde-3-phosphate dehydrogenase (GAPDH) primers, F 5'-TCCACCACCCTGTTGCTGTA-3', R 5'-ACCACAGTCCATGCCATCAC-3'.

Telomerase assay. Telomerase activity was assayed with the PCR-based modified, telomeric repeat amplification protocol (TRAP) assay (13). Each cell pellet $\left(1 \times 10^{5}\right)$ was resuspended in $200 \mu 1$ cold TRAP lysis buffer, incubated for $30 \mathrm{~min}$ on ice, and centrifuged at $15,000 \times \mathrm{g}$ for $20 \mathrm{~min}$ at $4^{\circ} \mathrm{C}$. The extract equivalent of 10 cells $(2 \mu \mathrm{l})$ was incubated with $48 \mu \mathrm{l}$ $20 \mathrm{mM}$ Tris- $\mathrm{HCl}$ (pH 8.3), $1.5 \mathrm{mM} \mathrm{MgCl}_{2}, 68 \mathrm{mM} \mathrm{KCl}$, $0.05 \%$ Tween-20, 1 mM EGTA, $50 \mathrm{mM}$ dNTP, $5 \mu \mathrm{g}$ BSA, $2 \mathrm{U}$ Taq DNA polymerase (Invitrogen), 10-attg internal telomerase assay standard DNA, and $0.1 \mu \mathrm{g}$ extra-purified TS primer (5'-AATCCGTCGTCGAGCAGAGTT-3') at $20^{\circ} \mathrm{C}$ for $30 \mathrm{~min}$ and then heated at $90^{\circ} \mathrm{C}$ for $3 \mathrm{~min}$. During the above step, $0.1 \mu \mathrm{g}(2 \mu \mathrm{l})$ of extra-purified CXII primer (5'-CCCTTACCC TTACCCTTACCCT-3') was added, and the reaction mixture was subjected to $31 \mathrm{PCR}$ cycles at $94^{\circ} \mathrm{C}$. Electrophoresis was performed on $12 \%$ nondenaturing acrylamide gels, stained with SYBR-Green (FMC Bioproducts, Rockland, ME), and bands were visualized using a chemiluminescence image analyzer.

Immunohistochemistry. Cells were cultured in $35-\mathrm{mm}^{2}$ culture dishes until subconfluent, then fixed with $3.5 \%$ neutral buffered formalin for $1 \mathrm{~h}$ and washed 3 times with phosphate-buffered saline (PBS, $145 \mathrm{mM} \mathrm{NaCl}, 2.8 \mathrm{mM} \mathrm{NaH}_{2} \mathrm{PO}_{4}, 7.2 \mathrm{mM}$ $\mathrm{Na}_{2} \mathrm{HPO}_{4}, \mathrm{pH}$ 7.2). They were treated with $0.3 \%$ hydrogen peroxide in $100 \%$ methanol for $20 \mathrm{~min}$, and washed 3 times with PBS. Immunostaining was performed using the Envision $^{+}$system (DakoCytomation, Carpinteria, CA, USA). Primary antibodies against cytokeratin monoclonal antibodies (AE1/AE3, Biomeda and CAM5.2, Becton Deckinson immunocytometry system), vimentin monoclonal antibody (1:200, DakoCytomation), lactoferrin polyclonal antibody (1:200, DakoCytomation), S-100 protein polyclonal antibody (1:200, DakoCytomation), $\alpha$-smooth muscle actin monoclonal antibody (1:200, DakoCytomation) and calponin monoclonal antibody (1:100, DakoCytomation) were applied at suitable dilutions and incubated overnight at $4^{\circ} \mathrm{C}$. Diaminobenzidinetetrahydrochloride was used as a chromogen and Mayer's hematoxylin was used as a counterstain.

Immunofluorescence double staining. Cells were fixed with $10 \%$ neutral buffered formalin for $20 \mathrm{~min}$ at room temperature. To block non-specific binding, normal goat serum was applied for $30 \mathrm{~min}$, and cells were then rinsed in $0.2 \%$ Triton X-100 in $0.01 \mathrm{M}$ PBS for $5 \mathrm{~min}$. Primary antibodies against cytokeratin polyclonal antibody (1:100, DakoCytomation), anti-vimentin monoclonal antibody (1:100, DakoCytomation), anti-ZO-1 monoclonal antibody (1:100, Zymed Laboratories, South San Francisco, CA) and anti-claudin-1 polyclonal antibody (1:100, Zymed) were applied at room temperature for $1 \mathrm{~h}$. After washing, anti-rabbit IgG Alexa fluor ${ }^{\circledR} 488$ (1: 200, Molecular Probes, Eugene, OR) and anti-mouse IgG Alexa fluor ${ }^{\circledR} 568$ (1: 200, Molecular Probes) were applied at room temperature for $1 \mathrm{~h}$. TO-PRO ${ }^{\circledR}-3$ iodide (1:500, Molecular Probes) was used for nuclear counterstaining. The staining was observed using conventional fluorescence microscopy (Axiophot 2, Carl Zeiss, München-Hallbergmoos, Germany).

Ultrastructural study. Cells were fixed with modified Karnovsky fixative (2\% paraformaldehyde buffer and $2.5 \%$ glutaraldehyde in $0.2 \mathrm{M}$ cacodylate buffer) for $30 \mathrm{~min}$ at room temperature, and then were collected as cell pellets. Samples were post-fixed with $1 \%$ osmium in $0.2 \mathrm{M}$ cacodylate buffer, dehydrated and embedded in EPON812 (TAAB Laboratories Equipment, Berkshire, UK). Ultra-thin sections were cut and double stained with uranyl acetate and lead citrate. Specimens were examined by transmission electron microscopy (H-7100, Hitachi, Tokyo, Japan).

\section{Results}

Histology of PA and cytology of PA cells. The tumor showed typical histological features of PA including an admixture of epithelial and mesenchymal-like components such as those found in myxoid and chondroid tissues (Fig. 1A). In culture, 
A

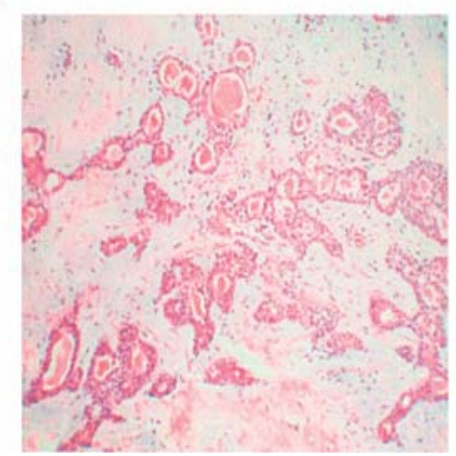

B

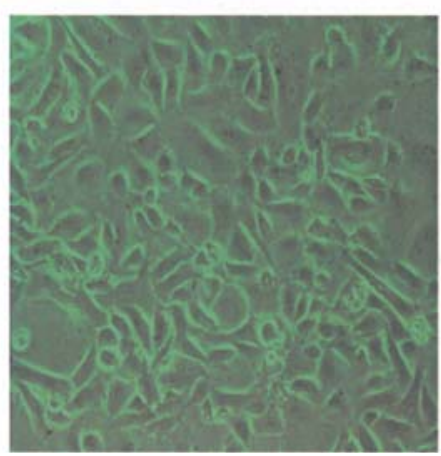

Figure 1. (A) Histological appearance of the original PA tumor in situ. The tumor consisted of epithelial and mesenchymal-like components such as those found in myxoid and chondroid tissues. (B) Morphology of non-transfected PA parental cells in culture. Cells were small and round or short and spindle-shaped.

A

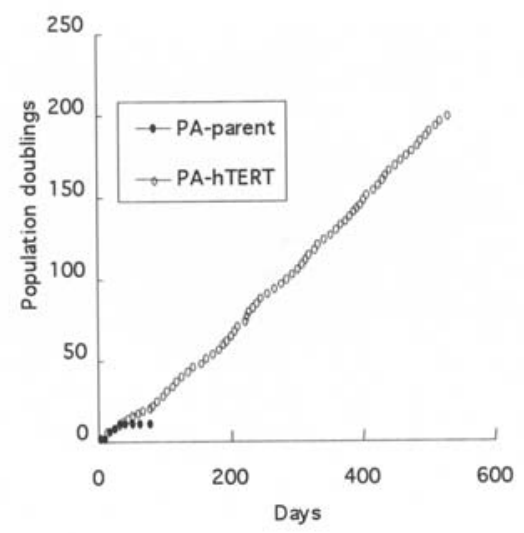

C
B

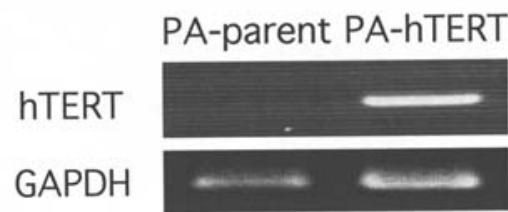

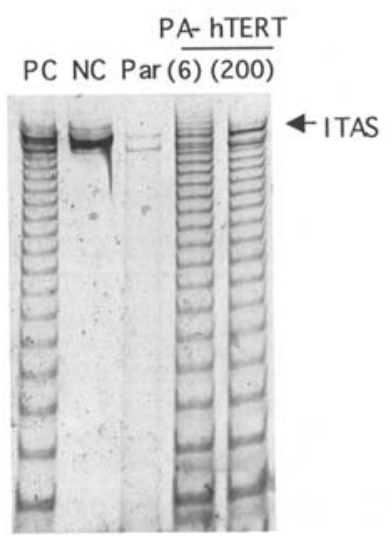

Figure 2. (A) PDLs of non-transfected PA-parent cells and hTERT-transfected PA (PA-hTERT) cells. PA-parent cells stopped growing after 6 PDLs, but PAhTERT cells were immortalized and grew over 200 PDLs. (B) Expression of the hTERT gene by RT-PCR analysis in PA-parent cells and PA-hTERT cells. High expression of the hTERT gene was observed in PA-hTERT cells but not in PA-parent cells. (C) Telomerase activity of PA-parent cells and PA-hTERT cells was determined by the TRAP assay. Telomerase activity was present in PA-hTERT cells, but not in PA-parent cells. The internal control consists of a 10-attg internal telomerase standard (ITAS) DNA. PC, positive control (extract of an oral cancer cell line, HSC2); NC, negative control, without cell extract; Par, parental cells. Cells at 6 and 200 PDLs were used.

the appearance of PA cells included small round or short spindle-shaped cells (Fig. 1B).

Immortalization of PA cells. PA cells transfected with hTERT (PA-hTERT) bypassed senescence and grew over 200 PDLs without significant growth retardation (Fig. 2A). In contrast, non-transfected PA parental cells entered crisis at about 6 PDLs. We confirmed the high expression of hTERT in PAhTERT cells, but not in parental cells by RT-PCR analysis (Fig. 2B). Although parental cells did not express telomerase activity, PA-hTERT maintained its telomerase activity even after 200 PDLs (Fig. 2C). 
A

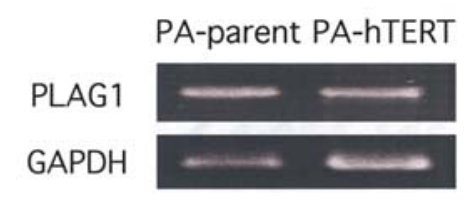

B
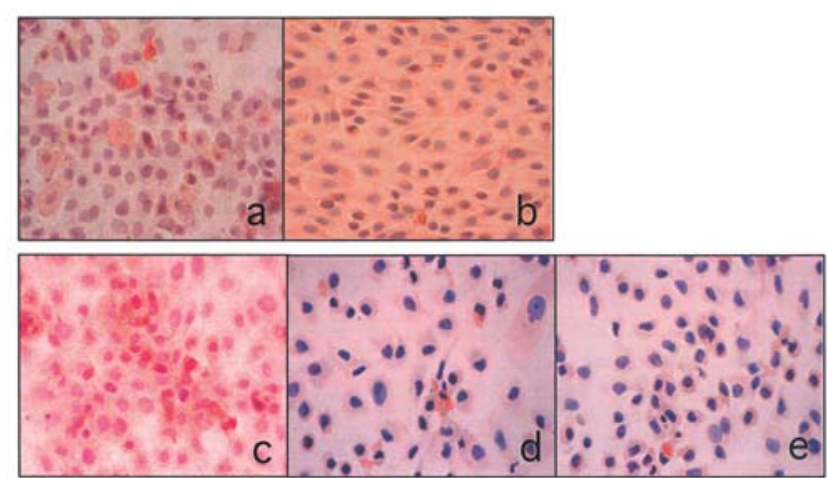

Figure 3. (A) Expression of the PA1 (PLAG1) gene by RT-PCR in PA-parent cells and in PA-hTERT cells. Both cell lines expressed PLAG1 mRNA at the same level. (B) Immunohistochemical staining. PA-hTERT was strongly positive for cytokeratin antibody (a), vimentin antibody (b), LF antibody (c) and calponin (d), and S-100 protein (e) was weakly positive.

Expression of PLAG1 mRNA. To identify cells derived from PA, we examined the expression of PLAG1 mRNA using RT-PCR. PLAG1 is a zinc finger transcription factor gene, which is consistently rearranged and overexpressed in human PA of the salivary glands with $8 \mathrm{q} 12$ translocations $(14,15)$. Parental PA cells and PA-hTERT cells expressed PLAG1 at the same level (Fig. 3A).

Immunohistochemical staining. The morphologic appearance of PA-hTERT cells was small and round or short and spindleshaped, identical to the appearance of parental PA cells. Immunohistochemically, most PA-hTERT cells were strongly positive for cytokeratin (Fig. 3Ba) and vimentin (Fig. 3Bb). In addition, some cells were intensely positive for lactoferrin (Fig. 3Bc), $\alpha$-smooth muscle actin or calponin (Figs. 3Bd). S-100 protein positive cells were also seen occasionally (Fig. 3Be).

Immunofluorescence double staining. Coexpression of cytokeratin and vimentin was distinct in the cytoplasm of PAhTERT cells. Vimentin (red color) was distributed in the central areas of the cells surrounding the nuclei, while cytokeratin (green color) was distributed throughout the cytoplasm including peripheral areas. Nuclei were counterstained as dark red by TO-PRO (Fig. 4A). Also tight junction proteins were found in PA-hTERT cells. ZO-1 was observed at the cellto-cell contact area clearly, and also with diffuse and weak staining in the cytoplasm (Fig. 4B). Coexpression of ZO-1 and claudin-1 was observed at the peripheral cell process. Expression of claudin-1 was observed as a continuous line and ZO-1 showed punctiform. Abundant expression of ZO-1 and claudin-1 were in the cytoplasm. Nuclei were indicated as dark red. (Fig. 4C)
A

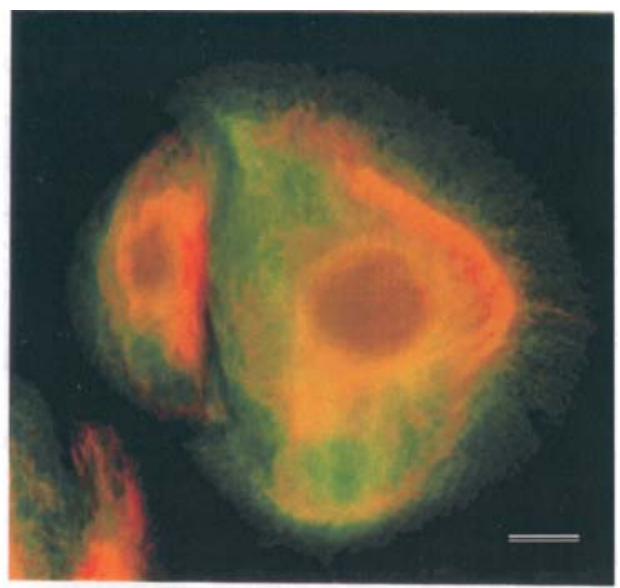

B

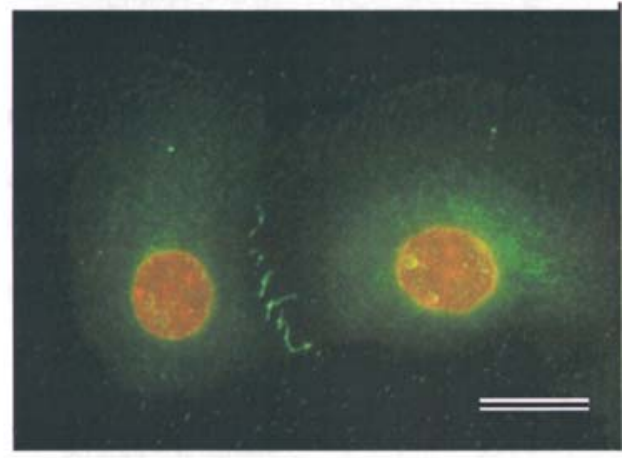

C

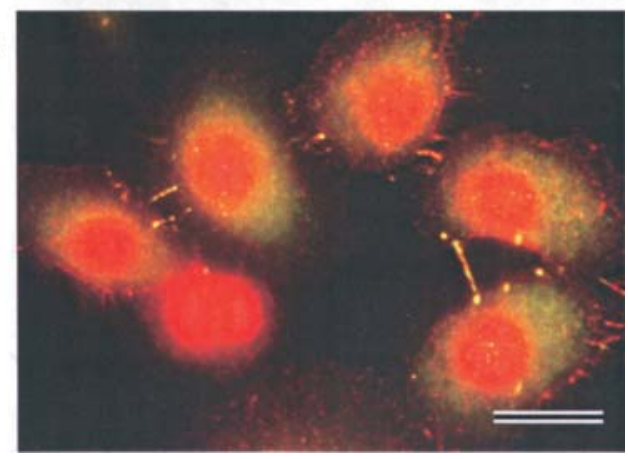

Figure 4. (A) Cytokeratin (green color) and vimentin (red color) were coexpressed distinctly in the cytoplasm. Vimentin was mainly distributed surrounding the nuclei, while cytokeratin was localized over the entire cytoplasm including the peripheral area (bar, $10 \mu \mathrm{m}$ ). (B) Expression of ZO-1 was observed distinctly at the cell-to-cell area discontinuously (original magnification x972; bar, $15.4 \mu \mathrm{m}$ ). (C) Coexpression of claudin-1 (red) and ZO-1 (green) was found at the peripheral cell process. While expression of claudin-1 was linear and continuous, ZO-1 showed punctiform. Both of them were also observed weakly and diffusely in cytoplasm. Nuclei were counterstained as dark red by TO-PRO (original magnification x680; bar, $22.0 \mu \mathrm{m})$.

Ultrastructural findings. Ultrastructurally, cytoplasmic organelles, including many bulging mitochondria, rough endoplasmic reticulum and Golgi apparatus, were recognized clearly. Many bundles of intermediate filaments and forming tonofilaments were observed surrounding the nuclei, and numerous microfilaments were localized in marginal areas of the cytoplasm and attached surfaces (Fig. 5A). A few incomplete amorphous, low-density secretory-like granules were also found, and some coated vesicles but not caveolae were aligned at the periphery of the plasma membrane (Fig. 5B). Golgi apparatus was also observed near the nuclei. At the cell junctional area, rich actin filaments were aligned 


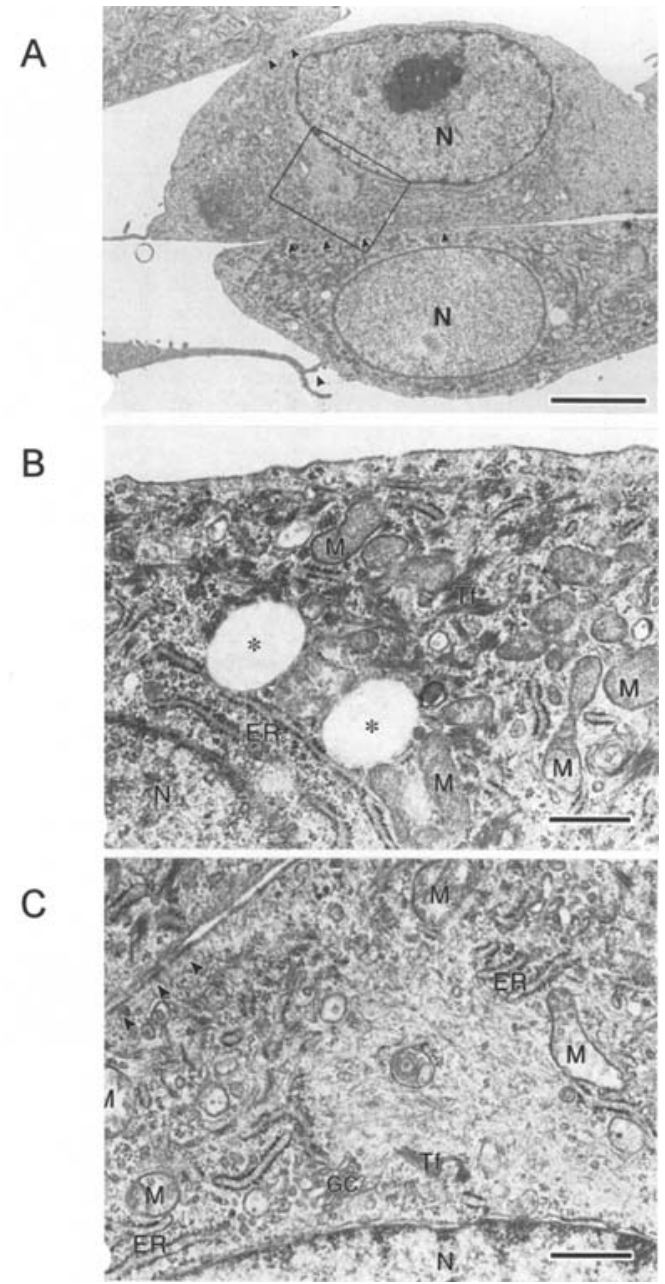

Figure 5. (A) Abundant cytoplasmic organelles and intermediate filaments were observed surrounding the nuclei (N) (bar, $3 \mu \mathrm{m}$ ). (B) Cytoplasmic organelles, rough endoplasmic reticulum (ER) and many bulging mitochondria (M) were recognized between the many bundles of tonofilaments (Tf). ${ }^{*}$ A few incomplete amorphous low-density secretory-like granules were recognized. Coated vesicles but no caveolae were aligned at the periphery of the plasma membrane. (C) Golgi apparatus (GC) was also observed near the nuclei (N). At the cell junctional area, rich actin filaments were aligned beneath the plasma membrane, and adherence junctions or a desmosome-like structure (arrow head) were recognized (bar, $0.5 \mu \mathrm{m}$ ).

beneath the plasma membrane, and adherence junctions or a desmosome-like structure were recognized (Fig. 5C).

\section{Discussion}

PA, the most common type of salivary gland tumor, shows unique histopathologic features, i.e., a mixture of epithelial and mesenchymal-like components normally seen in myxoid or chondroid tissues. The presence of both epithelial and mesenchymal-like components has led to considerable discussion about the histogenesis of this tumor. At the present time, it has been widely accepted that PA is a purely epithelial tumor and that its mesenchymal-like components are composed of neoplastic myoepithelial cells and their products, such as mucopolysaccharide matrix, elastic fiber and basement membrane-like materials, based on ultrastructural and immunohistochemical analyses $(1,2)$. However, the mechanism by which neoplastic myoepithelial cells form the mesenchymal- like components is not yet clarified, in part because there is no suitable in vitro model for PA. Therefore, the aim of this study was to create immortalized cells from PA that can grow stably and maintain the cellular properties of PA to allow analyses of their characteristic patterns of differentiation.

It is difficult to establish immortalized cells originating from benign tumors because of their slow growing nature and quick senescence in culture. Therefore, to prevent their senescence and to immortalize cell lines, some researchers use oncogenes or genes that are connected with immortalization, such as SV40, HPVE6/E7 and hTERT (16-19). However, SV40 and HPVE6/E7 may alter the nature of cells such as their contact inhibition and cell morphology (20). It has been reported that expression of hTERT and subsequent telomerase activity successfully immortalizes normal cells and benign neoplastic cells without otherwise altering the parent cells $(18,19,21-23)$. In the present study, transfection with the hTERT gene immortalized cells from PA, a benign salivary tumor. Expression of the mRNA for the hTERT gene and telomerase activity as measured by the TRAP assay were recognized in the transfected PA-hTERT cells, but not in the parental PA cells. PA-hTERT cells survived beyond normal programmed senescence over 200 PDLs, which indicates that a stable transfection occurred. The extended life span of these cells makes them useful for studying mechanisms involved in the development and differentiation of PA. Both the PAhTERT cells and parental PA cells showed small rounded or short spindle-shaped morphology and they expressed PLAG1 mRNA. PA-hTERT cells were positive for cytokeratins, a marker of epithelial cells, which confirmed their derivation from parenchymal cells, not from the stromal cells. In addition, immunohistochemical analyses revealed that PA-hTERT cells are composed of secretory epithelial cells positive for lactoferrin and myoepithelial cells positive for $\alpha$-smooth muscle actin and calponin.

A few incomplete amorphous, low-density secretory-like granules were recognized, and many bundles of intermediate filaments forming tonofilaments and microfilaments were also observed in the cytoplasm of the cells, ultrastructurally. Furthermore, adherence junctions were also observed between the adjacent cells, and coexpression of ZO-1 and claudin-1 tight junction proteins was observed at the cell-to-cell contact area and peripheral cell process by double immunofluorescence staining. Tight junctions form well-developed networks of continuous intra-membranous strands, which consist of tight junction molecules, occluding (24), claudins (25) and peripheral membrane proteins including ZO-1 (26). Important roles of tight junctions were as barriers within intercellular spaces, and fences determining a boundary line between apical and basolateral plasma membranes, in addition to the maintenance of cellular polarity (27). From this study, it is not clear whether pleomorphic adenoma reveals only one single cell type with a myoepithelial character or whether there are different phenotypes. Studies are needed to explore the function of one cell in detail.

Hence, PA-hTERT cells are a mixed population of secretory epithelial cells and neoplastic myoepithelial cells similar to those of PA in vivo. These findings indicate that PA-hTERT cells, immortalized by transfection with hTERT, maintain their original phenotypic characteristics. 
Overall, these findings suggest that these immortalized PA-hTERT cells will be useful for analyzing the mechanisms that regulate the differentiation of PA.

\section{Acknowledgements}

This study was supported in part by grants-in-aid from the Ministry of Education, Science and Culture of Japan.

\section{References}

1. Takeuchi J, Sobue M, Yoshida M, Esaki T and Katoh Y: Pleomorphic adenoma of the salivary gland with special reference to histochemical and electron microscopic studies and biochemical analysis of glycosaminoglycans in vitro. Cancer 36: 1771-1789, 1975.

2. Zhao M, Takata T, Kudo Y, et al: Biosynthesis of glycosaminoglycans and aggrecan by tumor cells in salivary pleomorphic adenoma: ultrastructural evidence. J Oral Pathol Med 28: 442-450, 1999.

3. Harley CB, Kim NW, Prowse KR, et al: Telomerase, cell immortality, and cancer. Cold Spring Harb Symp Quant Biol 59: 307-315, 1994.

4. Meyerson M, Counter CM, Eaton EN, et al: hEST2, the putative human telomerase catalytic subunit gene, is upregulated in tumor cells and during immortalization. Cell 90: 785-795, 1997.

5. Nakamura TM, Mori GB, Chapman KB, et al: Telomerase catalystic subunit homologs from fission yeast and human, Science 277: 955-959, 1997.

6. Nakayama J-I, Tahara H, Tahara E, et al: Telomerase activation by TRT in human normal fibroblasts and hepatocellular carcinomas. Nat Genet 18: 65-68, 1998.

7. Ulner GA, Hu J-F, Vu TH, Giudice LC and Hoffman AR: Telomerase activity in human development is regulated by human telomerase reverse transcription and by alternate splicing of hTERT transcripts. Cancer Res 58: 4168-4172, 1998.

8. Bodnar AG, Ouellette M, Frolkis M, et al: Extension of lifespan by introduction of telomerase into normal human cells. Science 279: 349-352, 1998.

9. Ramakrishnan S, Eppenberger U, Muller H, Shinkai Y and Narayanan R: Expression profile of the putative catalytic subunit of the telomerase gene. Cancer Res 58: 622-625, 1998.

10. Meyerson M: Role of telomerase in normal and cancer cells: J Clin Oncol 18: 2626-2634, 2000.

11. Ulaner GA, Hu-J-F, Vu TH, Oruganri H, Giuduce LC and Hoffman AR: Regulation of telomerase by alternate splicing of human telomerase reverse transcriptase (hTERT) in normal and neoplastic ovary, endometrium, and myometrium. Int J Cancer 85: $330-335,2000$.
12. Zheng PS, Iwasaka T, Yamasaki F, et al: Telomerase activity in gynecologic tumors. Gynecol Oncol 64: 171-175, 1997.

13. Kim NW, Piatyszek MA, Prowse KR, et al: Specific association of human telomerase activity with immortal cells and cancer. Science 266: 2011-2015, 1994.

14. Kas K, Voz ML, Roijer E, Astrom AK, Meyen E, Stenman G and Van de Ven WJ: Promoter swapping between the genes for a novel zinc finger protein and beta-catenin in pleomorphic adenomas with $\mathrm{t}(3 ; 8)(\mathrm{p} 21 ; \mathrm{q} 12)$ translocations. Nat Genet 15: 170-174, 1997.

15. Kas K, Voz ML, Hensen K, Meyen E and Van de Ven WJ: Transcriptional activation capacity of the novel PLGA family of zinc finger proteins. J Biol Chem 273: 23026-23032, 1998.

16. Jat PS and Sharp PA: Large T antigens of simian virus 40 and polyomavirus efficiently establish primary fibroblasts. J Virol 59: 746-750, 1986.

17. Munger K and Howley PM: Human papillomavirus immortalization and transformation functions. Virus Res 89: 213-228, 2002.

18. Shay JW, Wright WE and Werbin H: Defining the molecular mechanisms of human cell immortalization. Biochim Biophys Acta 1072: 1-7, 1991

19. Carney AS, Tahara H, Swartz CD, et al: Immortalization of human uterine leiomyoma and myometrial cell lines after induction of telomerase activity: molecular and phenotypic characteristics. Lab Invest 82: 719-728, 2002.

20. Yoo GH, Washington J, Oliver J, et al: The effects of exogenous p53 overexpression on HPV-immortalized and carcinogen transformed oral keratinocytes. Cancer 94: 159-166, 2002.

21. Counter CM, Hahn WC, Wei W, et al: Dissociation among in vitro telomerase maintenance, and cellular immortalization. Proc Natl Acad Sci USA 95: 14723-14728, 1998.

22. Halvorson TL, Leibowitz G and Lexine F: Telomerase activity is sufficient to allow transformed cells to escape from crisis. Mol Cell Biol 19: 1864-1870, 1999.

23. Colgin LM and Reddel RR: Telomere maintenance mechanisms and cellular immortalization. Curr Opin Genet Dev 9: 97-103, 1999.

24. Furuse M, Hirase T, Itoh M, Nagafuchi A, Yonemura S, Tsukita S and Tsukita S: Occludin: a novel integral membrane protein localizing at tight junctions. J Cell Biol 123: 1777-1788, 1993.

25. Furuse M, Fujita K, Hiiragi T, Fujimoto K, Tsukita S and Tsukita S: Claudin-1 and -2: novel integral membrane proteins localizing at tight junctions with no sequence similarity to occludin. J Cell Biol 141: 1539-1550, 1998.

26. Stevenson BR, Siliciano JD, Mooseker MS and Goodenough DA: Identification of ZO-1: a high molecular weight polypeptide associated with the tight junction (zonula occludens) in a variety of epithelia. J Cell Biol 103: 755-766, 1986.

27. Tsukita $S$ and Furuse M: Occludin and claudins in tight-junction strands: leading or supporting players? Trends Cell Biol 9: 268-273, 1999. 